\title{
On the thresholds, probability densities, and critical exponents of Bak-Sneppen-like models
}

\author{
Guilherme J. M. Garcia* and Ronald Dickman ${ }^{\dagger}$ \\ Departamento de Física, Instituto de Ciências Exatas \\ Universidade Federal de Minas Gerais, Caixa Postal 702 \\ CEP 30123-970, Belo Horizonte - Minas Gerais, Brazil
}

(August 22, 2018)

\begin{abstract}
We report a simple method to accurately determine the threshold and the exponent $\nu$ of the Bak-Sneppen model and also investigate the BS universality class. For the random-neighbor version of the BS model, we find the threshold $x^{*}=0.33332(3)$, in agreement with the exact result $x^{*}=1 / 3$ given by mean-field theory. For the one-dimensional original model, we find $x^{*}=0.6672(2)$ in good agreement with the results reported in the literature; for the anisotropic BS model we obtain $x^{*}=0.7240(1)$. We study the finite size effect $x^{*}(L)-x^{*}(L \rightarrow \infty) \propto L^{-\nu}$, observed in a system with $L$ sites, and find $\nu=1.00(1)$ for the random-neighbor version, $\nu=1.40(1)$ for the original model, and $\nu=1.58(1)$ for the anisotropic case. Finally, we discuss the effect of defining the extremal site as the one which minimizes a general function $f(x)$, instead of simply $f(x)=x$ as in the original updating rule. We emphasize that models with extremal dynamics have singular stationary probability distributions $p(x)$. Our simulations indicate the existence of two symmetry-based universality classes.
\end{abstract}

PACS: 05.65.+b, 02.30.Ks, 05.40.-a, 87.10.+e

keywords: Bak-Sneppen model; threshold; finite-size scaling; anisotropic BS model; critical exponents; universality;

* Electronic address: gjmg@fisica.ufmg.br

$\dagger$ Electronic address: dickman@fisica.ufmg.br

Corresponding author: Ronald Dickman

telephone: 55-31-3499-5665

FAX: 55-31-3499-5600 


\section{INTRODUCTION}

Power-law distributions are often found in natural and social systems. Examples include the distributions of rain event sizes and durations [1-4], earthquake sizes [5,6], internet connections [7], and food webs [8]. Why do such diverse phenomena exhibit scale invariance, as reflected in power-law distributions? A concept introduced by Bak and collaborators to account in part for scale-invariance in nature is self-organized criticality (SOC), which asserts that "systems that are far from equilibrium evolve to a dynamical attractor poised at criticality" [9].

Over the last two decades, many models exhibing self-organized criticality have been proposed: sandpile models $[6,10,11]$, the OFC model for earthquakes [12], forest fire models [13], the ballistic deposition model of rough surfaces [14], and the Bak-Sneppen evolution model [15]. Many SOC models are defined in such a manner that they are trapped at criticality [16]. In particular, sandpile models are maintained at the critical point by "slow driving in a system exhibiting an absorbing-state phase transition with a conserved density" [16]. A different path to SOC is so-called extremal dynamics. In this case, the system is critical provided that an 'extremal condition' is respected [16-18].

The Bak-Sneppen evolution model is one of the simplest models exhibiting SOC via extremal dynamics. For this reason, it has atracted much attention in the statistical physics community and has been studied through various approaches, including simulation [19,21,22], theoretical analysis [23-25], probabilistic analysis (run time statistics) [26,27], renormalization group [28,29], field theory [30] and mean-field theory [16-18,31]. Some variants have been proposed, for example the anisotropic BS model [32,33].

In this paper, we investigate the scaling behavior of the Bak-Sneppen model. We study the finite size scaling $x_{L}^{*}-x_{\infty}^{*}=k L^{-\nu}$, where $x_{L}^{*}$ is the threshold for a one-dimensional system of $L$ sites and $k$ is a constant. This provides an accurate estimate of the threshold $x_{\infty}^{*}$ and of the exponent $\nu$. We also discuss which modifications of the updating rule preserve the universality class. In agreement with Ref. [18], we find that extremal dynamics leads to a singular stationary distribution $p(x)$, and that models with symmetric updating rules belong to the same universality class of the original model. The specific form of the distribution $p(x)$ is, however, nonuniversal.

The balance of this paper is organized as follows. In Sec. II, we define the BS model along with the random-neighbor and the anisotropic BS versions. We define also a "cosine BS model". We calculate the threshold and the exponent $\nu$ of the first three models in Sec. III. In Sec. IV, we study the BS universality class. We summarize our conclusions in Sec. V.

\section{MODELS}

The Bak-Sneppen model [15] is defined on a d-dimensional lattice with $L^{d}$ sites and periodic boundaries. Each site bears a real-valued variable $x_{i} \in[0,1)$. 
At time zero, all the $x_{i}$ are assigned independent random values drawn from a distribution uniform on $[0,1)$. At each step of the evolution, the site $x_{m}$, that is the global minimum of the $\left\{x_{i}\right\}$, along with its $2 d$ nearest neighbors, are replaced by new random numbers taken from the same distribution. This process is repeated indefinitely.

In the random-neighbor version of the model, all sites are considered neighbors and, at each time step, the site $m$ along with two randomly chosen neighbors are substituted with new random numbers. All the other definitions remain the same. A further variant of the model is the anisotropic BS model. In this case, only the site $m$ and its right neighbor are replaced at each time step. We also study a modified Bak-Sneppen model, that differs from the original in the way the site $x_{m}$ is identified. We baptize this the "cosine BS model". Instead of minimizing $f(x)=x$ as in the original model, $x_{m}$ minimizes $f(x)=\cos (2 n \pi x)$ for some integer $n$, so that $f$ has $n$ minima on the interval $[0,1]$. Finally, in order to investigate the effect of symmetry in the updating rule, we define an "anisotropic cosine BS model", in which we have the spatial anisotropy of the anisotropic BS model and the extremal site defined as in the cosine BS model.

\section{DETERMINING THE THRESHOLD}

Simulation of the BS model shows that, after a transient time, the system achieves a statistically stationary state in which the density of $x_{i}$ vanishes for $x$ less than a certain threshold $x^{*}$ and is uniform above $x^{*}$. In other words, for an infinite system and in the stationary state, the distribution $p(x)$ is the step-fuction

$$
p(x)=\left\{\begin{array}{cc}
0, & x<x^{*} \\
C, & x>x^{*}
\end{array} .\right.
$$

This singular probability density is associated with scaling properties reminiscent of a critical point; the upper critical dimension $d_{c}=4$ [22]. The Bak-Sneppen model does not appear to belong to any of the known universality classes of critical phenomena, and may be said to define its proper class.

Accurate determinations of $x^{*}$ were reported by Grassberger [19] and Paczuski and coworkers [9], who found $x^{*}=0.66702(8)$ and $x^{*}=0.66702(3)$, respectively. We introduce here a third method to determine $x^{*}$. For a finite system (see Fig. 1), the distribution $p(x)$ is continuous, i.e., the step function singularity is rounded [20]. Nevertheless, as is apparent from the figure, $p(x)=C_{L}$, constant, for $x$ well above the transition region. If $p(x)$ is a step function, normalization implies that $C\left(1-x^{*}\right)=1$. This suggests that we

define a finite-system threshold via $x_{L}^{*}=1-1 / C_{L}$. If we now assume that the Bak-Sneppen model obeys the finite-size scaling relation

$$
x_{L}^{*}-x_{\infty}^{*}=k L^{-1 / \nu},
$$


where $k$ is a constant and $x_{\infty}^{*}$ is the threshold for an infinite system, we have a simple method for determining the threshold of Bak-Sneppen-like models.

We simulate the Bak-Sneppen model, the anisotropic BS model, and the random-neighbor version on lattices of $L=63,125,250,500,1000,2000,4000$ and 8000 sites (see Figure 1). We estimate the probability density $p(x)$ on the basis of a histogram of barrier frequencies, dividing [0,1] into 100 subintervals. Histograms are accumulated after $N_{s t}$ time steps, as required for the system to relax to the stationary state. (We find that for the original and the anisotropic models $N_{s t}=10^{8}$ sufficient to reach the stationary states for even the largest system studied, while for the random-neighbor version $N_{s t}=10^{7}$ is sufficient.) We make the histogram every $L$ time steps in runs of $10^{9}$ steps total.

To test the accuracy of the method described above, we applied it to the random-neighbor version of the model, for which the exact result $x^{*}=1 / 3$ is known from mean-field theory [16-18,31]. Analyzing our data for $x_{L}^{*}$ vs. $L$ using Eq. (2), we find $x^{*}=0.33332(3)$ and $\nu=1.00(1)$. This results agrees with the mean-field theory prediction and validates our method. For the original BS model, we find $x^{*}=0.6672(2)$ and $\nu=1.40(1)$. (The power law fit is shown in Fig. 2.) In accord with this are the results $x^{*}=0.66702(8)$ reported by Grassberger [19], $x^{*}=0.66702(3)$ found by Paczuski and collaborators [9], and $\nu=1.36$ [34]. Finally, for the anisotropic BS model, we obtain $x^{*}=$ $0.7240(1)$ and $\nu=1.58(1)$. To our knowledge, this is the first result for the threshold and the exponent $\nu$ of the anisotropic BS model.

\section{THE BS UNIVERSALITY CLASS}

As noted in the Introduction, extremal dynamics is one path to a selforganized critical state. Two questions therefore arise: 1) What are the trademarks of extremal dynamics? and 2) What other models belong to the universality class of the Bak-Sneppen model? In order to answer these questions, Garcia and Dickman [18] studied the effects of updating via $x_{i}^{\prime}=x_{i}^{\alpha}$ (with $\alpha=1 / 2$ or 2 ), instead of replacing these variables with random numbers. They found that these updating rules lead to quite distinct stationary probability densities $p(x)$, which nonetheless exhibit one or more discontinuities. Another caracteristic shared by all the variants studied in [18] is that the extremal site always belongs to the "prohibited region" for which $p(x)=0$. Although $p(x)$ and consequently the threshold are nonuniversal, all variants studied belong to the universality class of the original model. Moreover, it has been shown $[33,35,36]$ that models that break the symmetry of the original model fall into the universality class of the anisotropic BS model.

A related question is the robustness of BS scaling under modifications of extremal dynamics. It is known that scale invariance is lost when the extremal condition is relaxed, for example by introducing a nonzero probability for choosing a nonextremal site to be updated [16-18]. In this context it is

interesting to note that updating the $M$ smallest barriers (with $M>1$ ), at each step changes the scaling properties drastically [37]. 
As representatives of a further broad class of variants of the Bak-Sneppen model, we study here the isotropic and the anisotropic cosine BS models proposed in Sec. II. We simulate these models (with $n=5$ ) on a lattice of $L=2000$ sites and find the distribution $p(x)$ shown in figure 3 for the isotropic case. (In the anisotropic version, a similar distribution is found.) It is natural to regard the rounding of the singularities as a finite-size effect. Thus we find evidence that these updating rules also lead to singular probabilty densities. We have also investigated the distribution $P_{J}(r)$ that sucessive updated sites are separated by a distance $r$. This obeys the power law $P_{J}(r) \sim r^{-\pi}$ with $\pi=3.23(2)$ in the original model [32], and $\pi=2.401(2)$ in the anisotropic BS model [33]. We performed simulations of the cosine BS models using $10^{8}$ time steps, yielding $\pi=3.2(1)$ for the symmetric updating rule and $\pi=2.38(3)$ for asymmetric updating rule. Therefore a dynamics that respects the symmetry of the original model (that is, spatial isotropy), and that does not introduce any new conserved quantity, leads to the same exponents as the original model. On the other hand, asymmetric dynamics leads to the anisotropic BS model universality class.

\section{CONCLUSIONS}

We verify the finite-size scaling relation $x_{L}^{*}-x_{\infty}^{*}=k L^{-\nu}$ in the BakSneppen model, and use it to obtain accurate values for the threshold and for the exponent $\nu$ of the original, anisotropic and random-neighbor versions. We also study the effect of defining the extremal site as the one that minimizes a function $f(x)$ with multiple minima. Simulations show that this does not affect the universality class and strengthens the result of Ref. [18] that extremal dynamics leads to singular probability densities. Finally, our results indicate that variants that preserve the spatial isotropy of the original model belong to

the same universality class, while models which break that symmetry fall into the anisotropic BS model universality class.

\section{ACKNOWLEDGMENTS}

We thank CNPq and CAPES, Brazil, for finacial suport. 


\section{REFERENCES}

[1] O. Peters, C. Hertlein, K. Christensen, Phys. Rev. Lett. 88 (2002) 018701.

[2] O. Peters, K. Christensen, Phys. Rev. E 66 (2002) 036120.

[3] R. Dickman, Phys. Rev. Lett. 90 (2003) 108701.

[4] S. Lovejoy, M. Lilley, N. Desaulnies-Soucy, D. Schertzer, Phys. Rev. E 68 (2003) 025301(R).

[5] B. Gutenberg, C.F. Richter, Bull. Seismol. Soc. Am. 34 (1994) 185.

[6] P. Bak, C. Tang, K. Wiesenfeld, Phys. Rev. Lett. 59 (1987) 381.

[7] R. Pastor-Satorras and A. Vespignani, Evolution and Structure of the Internet, Cambridge University Press, Cambridge, 2004.

[8] D. Garlaschelli, G. Caldarelli, L. Pietronero, Nature 423 (2003) 165.

[9] M. Paczuski, S. Maslov, P. Bak, Phys. Rev. E 53 (1996) 414.

[10] S. S. Manna, J. Stat. Phys. 59 (1990) 509.

[11] S. S. Manna, J. Phys. A 24 (1991) L363.

[12] Z. Olami, H.J.S. Feder, K. Christensen, Phys. Rev. Lett. 68 (1992) 1244.

[13] P. Bak, K. Chen, C. Tang, Phys. Lett. A 147 (1990) 297.

[14] A.-L. Barabsi, H.E. Stanley, Fractal Concepts in Surface Growth, Cambridge University Press, New York, USA, 1995.

[15] P. Bak, K. Sneppen, Phys. Rev. Lett. 71 (1993) 4083.

[16] R. Dickman, M.A. Muñoz, A. Vespignani, S. Zapperi, Braz. J. Phys. 30 (2000) 27.

[17] D. Head, Eur. Phys. J. B 17 (2000) 289.

[18] G.J.M. Garcia, R. Dickman, PHYSICA A, to appear.

[19] P. Grassberger, Phys. Lett. A 200 (1995) 277.

[20] For $x<x *$ the approach to the step function is algebraic with $L$, while for $x>x *$ the convergence is exponential [18].

[21] P.D. Rios, M. Marsili, M. Vendruscolo, Phys. Rev. Lett. 80 (1998) 5746.

[22] S. Boettcher, M. Paczuski, Phys. Rev. Lett. 84 (2000) 2267.

[23] R. Meester, D. Znamenski, J. Stat. Phys. 109 (2002) 987.

[24] W. Li, X. Cai, Phys. Rev. E 62 (2000) 7743.

[25] S.N. Dorogovtsev, J.F.F. Mendes, Y.G. Pogorelov, Phys. Rev. E 62 (2000) 295.

[26] G. Caldarelli, M. Felici, A. Gabrielli, L. Pietronero, Phys. Rev. E 65 (2002) 046101.

[27] M. Felici, G. Caldarelli, A. Gabrielli, L. Pietronero, Phys. Rev. Lett. 86 (2001) 1896.

[28] M. Marsili, Europhys. Lett. 28 (1994) 385.

[29] B. Mikeska, Phys. Rev. E 55 (1997) 3708.

[30] M. Paczuski, S. Maslov, P. Bak, Europhys. Lett. 27 (1994) 97.

[31] Y.M. Pismak, Phys. Rev. E 56 (1997) R1326.

[32] D.A. Head, G.J. Rodgers, J. Phys. A 31 (1998) 3977.

[33] S. Maslov, P. De Los Rios, M. Marsili, Y.-C. Zhang, Phys. Rev. E 58 (1998) 7141.

[34] M. Paczuski, S. Maslov and P. Bak, Europhys. Lett. 27 (1994) 97; ibid. 28 (1995) 295. 
[35] D.A. Head, G.J. Rodgers, J. Phys. A 31 (1998) 3977.

[36] G.J.M. Garcia, R. Dickman, to be published.

[37] A. S. Datta, K. Christensen, H. Jeldtoft Jensen, Europhys. Lett. 50 (2000) 162. 


\section{FIGURES}

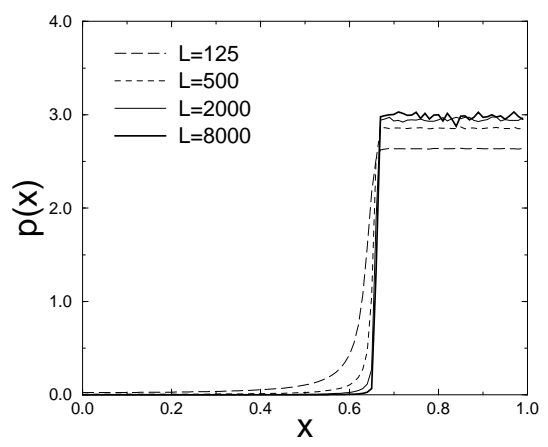

FIG. 1. Stationary density $p(x)$ for the original Bak-Sneppen model in one dimension, for various system sizes.

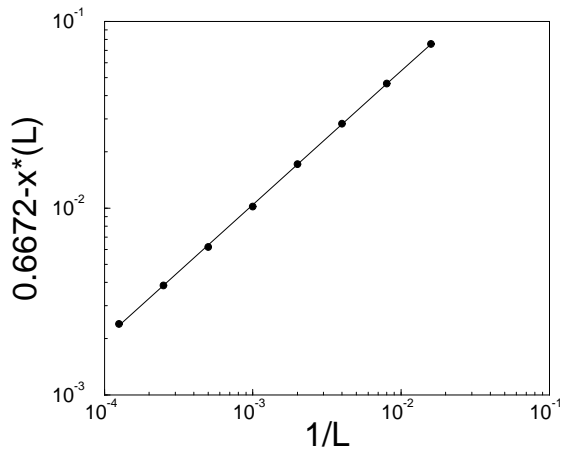

FIG. 2. Power law fit for $\left(x_{\infty}^{*}-x_{L}^{*}\right)$ vs. $1 / L$ for the original BS model. The slope of the straight line is 1.40 .

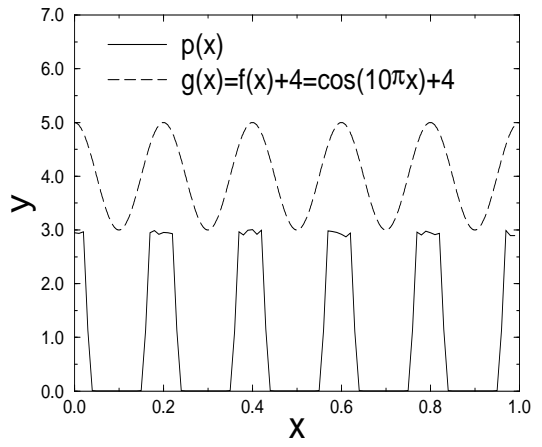

FIG. 3. Solid line: stationary density $p(x)$ for the cosine BS model $(n=5)$ for a system of $L=2000$ sites. Dashed line: function $f(x)=\cos (10 \pi x)$ that is minimized in the cosine BS model. ( $f(x)$ is shifted for better visualization.) For further details, see Section II. 